Proceedings of the 46th International School and Conference on the Physics of Semiconductors "Jaszowiec" 2017, Szczyrk

\title{
Response of Indium Antimonide at Sub-Terahertz and Terahertz Frequencies
}

\author{
D. Yavorskiy, K. Karpierz, M. Grynberg and J. Łusakowski* \\ Faculty of Physics, University of Warsaw, L. Pasteura 5, 02-093 Warsaw, Poland
}

\begin{abstract}
Responsivity of a bulk InSb detector at liquid helium temperature was studied in the frequency range $0.1 \mathrm{THz}<$ $f<2.5 \mathrm{THz}$. The detector was exposed to a monochromatic radiation and its response was registered as a function of magnetic field up to $7 \mathrm{~T}$. Due to a very strong magnetoresistance of InSb, a dependence of the photocurrent on magnetic field shows a totally different shape than that of the photoresistance. Thus, photoconductivity was found to be the only reliable way of data presentation. The detector develops a resonant response, related to the cyclotron resonance absorption, at $f \gtrsim 0.5 \mathrm{THz}$ but this response dominates the spectrum only at $f \gtrsim 1 \mathrm{THz}$. At $f \lesssim 1 \mathrm{THz}$ the response is mainly non-resonant and is caused by intra-band transitions. We conclude that an InSb detector can be used as a magnetic-field-tunable spectroscopic element only if one can assure that the frequency of photons which reach the detector is higher than about $1 \mathrm{THz}$.
\end{abstract}

DOI: 10.12693/APhysPolA.132.338

PACS/topics: THz spectroscopy. InSb THz detector

\section{Introduction}

Bulk indium antimonide crystals have been used for decades as detectors of $\mathrm{mm}$ and sub-mm waves [1]. There are many reasons for using such detectors in $\mathrm{THz}$ spectroscopy. First, they are fast because their response relies on electronic transitions. Second, since the detectors are cooled to liquid helium temperatures ( $4.2 \mathrm{~K}$ or less), they show a very low noise equivalent power and a high sensitivity. Third, their resonant response, based on electron transitions between Landau levels, allows to use such detectors as magnetic-field-tunable elements in far-infrared, or $\mathrm{THz}$, spectroscopic applications [2].

A successful construction and application of different types of Landau spectroscopy systems, in which InSb crystals were used either as sources or detectors of $\mathrm{THz}$ radiation [2], led to a misleading conviction that a response of InSb at sufficiently strong magnetic field $B$ (typically, above about $0.25 \mathrm{~T}$ ) must be caused by resonant inter-Landau-level transitions. The present paper shows that the reality is more complex. We have found that the response of InSb is a superposition of resonant (inter-Landau-level) and non-resonant (intraLandau-level) processes at any magnetic field and which transitions are dominant depends both on the frequency of radiation, $f$, and on $B$.

In available literature devoted to far-infrared spectroscopy on InSb, we have found only one trace of a study, presented below in Fig. 1, where a result similar to our data was described [1]. Under "similar" we mean a study of response of bulk $\mathrm{InSb}$ to radiation of sub- $\mathrm{THz}$ frequency and as a function of magnetic field. The figure shows that up $B=0.6 \mathrm{~T}$, an InSb detector responds in a broad range of frequency, coinciding with a sub- $\mathrm{THz}$

*corresponding author; e-mail: Jerzy.Lusakowski@fuw.edu.pl frequency band explored in the present study. This result imposes limits on application of InSb detector as a magnetic-field-tunable spectral device: observation of a detector's response at a quantizing magnetic field $B$ does not automatically mean that it is irradiated with photons of the cyclotron resonance (CR) frequency $f=e B / 2 \pi m$. Our study show that to assure such a correspondence, one has to be certain that the frequency of radiation is higher than about $1 \mathrm{THz}$.

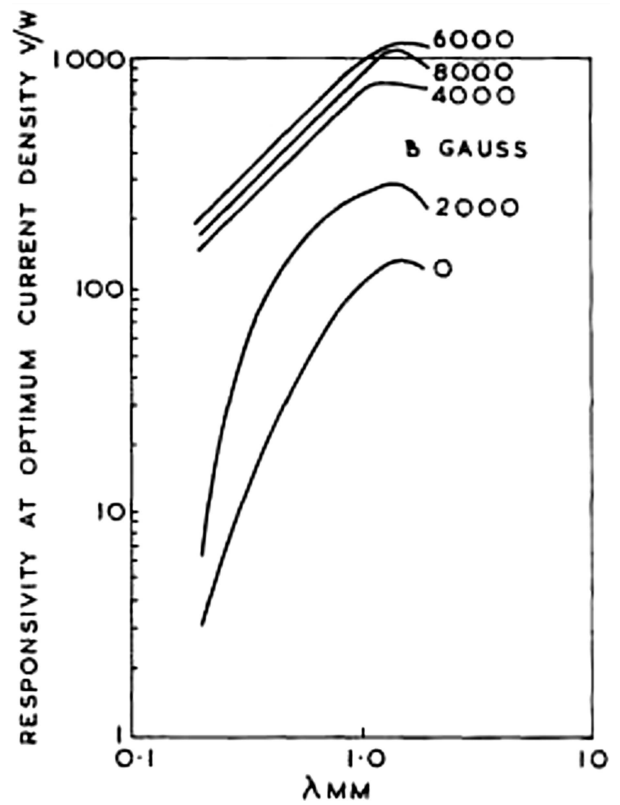

Fig. 1. Dependence of responsivity of an InSb detector upon wavelength and magnetic field at $1.8 \mathrm{~K}$ and at optimum current for each field. After Ref. [1].

\section{Experimental}

The detector used was a bulk InSb crystal in a meander shape attached to a wedged sapphire plate. The detector 
was placed in the center of a superconducting coil in a helium cryostat and cooled by an exchange gas at a stable temperature of about $4 \mathrm{~K}$. A monochromatic radiation was guided to the detector through a stainless steel tube, $12 \mathrm{~mm}$ in diameter. A cold filter placed in the tube screened the detector from thermal radiation of warm parts of the cryostat.

We used two sources of radiation. One of them was an optically pumped molecular laser which generated a few lines in the range 1-2.5 THz. The other source was a backward wave oscillator with a tunable fundamental frequency between 110 and $160 \mathrm{GHz}$. A set of frequency multipliers $(\times 2, \times 3$, and $\times 6)$ allowed us to cover most of the range between $0.1 \mathrm{THz}$ and $1 \mathrm{THz}$. The radiation was mechanically on/off chopped with a frequency of $170 \mathrm{~Hz}$ and a lock-in detection was used.

The signal measured was either a photoresistance (in this case, a current source supplied the detector with a small current of the order of $1 \mu \mathrm{A}$ and the lock-in was connected in parallel to the current source) or a photocurrent (in this case, the lock-in measured a voltage drop on a resistor connected in series with the detector, and a voltage source was used). The signal was registered as a function of magnetic field.

\section{Results and discussion}

We have found that a magnetic field dependence of the detector signal drastically depends on the measurement procedure. An example of this dependence, for the frequency of radiation equal to $0.7 \mathrm{THz}$, is presented in Fig. 2. The solid line represents the photocurrent which carries a signature of a cyclotron resonance transition at about $0.35 \mathrm{~T}$ and a broad structure resulting from nonresonant transitions. A dashed line shows results of the magnetoresistance measurements; it is totally different from the previous one, with a signature of the CR transition visible after magnification of a low- $B$ part of the spectrum, shown in the central inset. Both curves, after recalculation lead to the photoconductivity curve (thick dotted line) on which a cyclotron transition is only visible after magnification. A similar comparison (photocurrent vs. pthotoconductivity) at $2.5 \mathrm{THz}$ is shown in the left inset to Fig. 2.

A reason of such a huge difference between photocurrent and photoresistance is a very strong magnetoresistance of InSb which is shown in the inset to Fig. 2: the resistance of the detector increases between $2 \mathrm{k} \Omega$ to $1.2 \mathrm{M} \Omega$ when the magnetic field is increased to $3 \mathrm{~T}$. To take into account the magnetoresistance, we recalculated the raw data to the photoconductivity, which is presented as dashed lines in Fig. 2

The results presented in Fig. 2 indicate how one should interpret results of spectroscopic measurements with the $\mathrm{InSb}$ detector. Let us concentrate on the photoconductivity first (thick dotted line in Fig. 2). A non-resonant contribution to the photoconductivity is high at $B \approx 0$ and vanishes at about $0.5 \mathrm{~T}$, irrespective of the frequency of radiation. For InSb, the magnetic field of $0.5 \mathrm{~T}$ corresponds to the cyclotron resonance frequency of $1 \mathrm{THz}$.

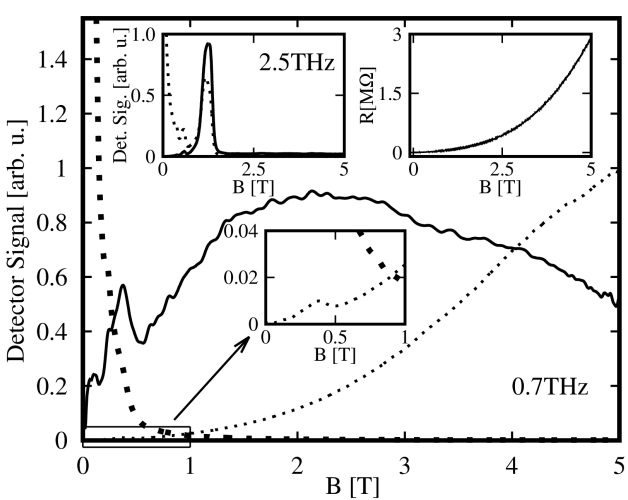

Fig. 2. A magnetic field dependence of photocurrent, photoresistance and photoconductivity (multiplied by 10) is presented with solid, thin dotted and thick dotted line, respectively, of the InSb detector at $0.7 \mathrm{THz}$. Right inset: a resistance of the detector as a function magnetic field; left inset: photocurrent (solid line) and photoconductivity (dotted line) of the detector at $2.5 \mathrm{THz}$ central inset: magnified low- $B$ part of the figure with a CR-related maximum visible in the photoresistance spectrum.

This means that for frequency greater than $1 \mathrm{THz}$, the $\mathrm{InSb}$ detector can be, for sure, used as a resonant and magnetic field-tunable spectroscopic device, but if one considers only the part of spectrum at $B>0.5 \mathrm{~T}$.

Next, the photocurrent appears to be the most sensitive to the cyclotron resonance, however, a single CRrelated line line can be observed only at $f>1 \mathrm{THz}$. For smaller $f$, the photocurrent is a mixture of resonant and non-resonant contributions, and - as it is shown in Fig. 2 - its non-zero value at high $B$ has nothing to do with resonant transitions.

\section{Conclusions}

We conclude that an InSb detector cannot be used to spectrally characterize unknown sources of $\mathrm{THz}$ radiation unless one is sure that the detected radiation is of a frequency higher than about $1.0 \mathrm{THz}$; this is of a particular importance if one relies on photocurrent or photoresistance data. If this is not certain, one can erroneously interpret a photocurrent or a photoresistance signal at high magnetic fields as resulting from a cyclotron resonance transitions, while it is due to a nonresonant response.

\section{Acknowledgments}

A financial support from a Polish National Science Centre UMO-2015/17/B/ST7/03630 grant is acknowledged.

\section{References}

[1] E.H. Puthley, Appl. Opt. 4, 649 (1965).

[2] W. Knap, D. Dur, A. Raymond, C. Meny, J. Leotin, S. Huant, B. Etienne, Rev. Sci. Instrum. 63, 3293 (1992). 